\title{
Systolic Anterior Motion of Mitral Valve Subchordal Apparatus: A Rare Echocardiographic Pattern in Non- Obstructive Hypertrophic Cardiomyopathy
}

\author{
Jezreel L. Taquiso a, b, Stephanie Martha O. Obillos ${ }^{\mathrm{a}}$, Joerelle V. Mojica ${ }^{\mathrm{a}}$, \\ Lauro L. Abrahan IV ${ }^{\text {a }}$, Elleen C. Cunanan ${ }^{a}$, Jaime Alfonso M. Aherrera ${ }^{\text {a }}$, \\ Jose Donato A. Magno ${ }^{a}$
}

\begin{abstract}
Systolic anterior motion (SAM) of the mitral valve or chordate is one characteristic seen in hypertrophic cardiomyopathy (HCM) either in obstructive or non-obstructive phenotypes. More often than not, the obstruction is caused by valvular rather than chordal SAM. We describe the role of echocardiography in identifying the actual anatomical location of the mitral valve apparatus involved in SAM and in assessing consequent left ventricular outflow tract (LVOT) obstruction in an otherwise asymptomatic patient. We report a case of a 29-year-old male admitted for an elective non-cardiac surgery, presenting with a cardiac murmur and left axis deviation with biventricular hypertrophy on electrocardiogram. On 2D transthoracic echocardiography (TTE), an asymmetrically hypertrophied left ventricle with systolic motion of anterior mitral valve was incidentally seen. Continuous wave Doppler assessment across the LVOT showed some gradient of obstruction (peak gradient: $9 \mathrm{~mm} \mathrm{Hg}$ ). Transesophageal echocardiography (TEE) demonstrated a redundant anterior mitral valve with the subchordal apparatus mainly causing SAM and confirmed the gradient obtained on TTE, with a mild degree, yet nonsignificant, degree of LVOT obstruction (mean gradient: $10 \mathrm{~mm} \mathrm{Hg}$ ) documented. Because of this finding, patient was cleared for surgery. Management was deemed conservative with emphasis on close surveillance for signs and symptoms attributable to development of significant LVOT obstruction in patients with HCM. To our knowledge, this is the first reported case in our country of an echocardiographic pattern of systolic anterior motion primarily of the subchordal mitral valve apparatus causing some, though non-significant, degree of LVOT obstruction in HCM. Echocardiographic features such as asymmetric left ventricular hypertrophy and presence of some LVOT obstruction caused primarily by subchordal apparatus could impact management in asymptomatic patients.
\end{abstract}

Manuscript submitted September 18, 2017, accepted September 27, 2017

aSection of Cardiology, Department of Medicine, University of the Philippines Manila - Philippine General Hospital, Manila, Philippines

${ }^{\mathrm{b}}$ Corresponding Author: Jezreel L. Taquiso, Section of Cardiology, Department of Medicine, University of the Philippines Manila - Philippine General Hospital, Taft Avenue 1000, Manila, Philippines.

Email: hanz2joe@gmail.com

doi: https://doi.org/10.14740/cr614w
Keywords: Subchordal systolic anterior motion; Hypertrophic cardiomyopathy

\section{Introduction}

Systolic anterior motion (SAM) of the mitral valve or chordate is one characteristic seen in hypertrophic cardiomyopathy (HCM) with flow obstruction. More often than not, the obstruction is caused by valvular rather than chordal SAM [1]. Systolic motion primarily by the chordae tendinae and subsequent left ventricular outflow tract (LVOT) obstruction are described mainly from case reports and small case series. Transesophageal echocardiography and (TEE) cine cardiac magnetic resonance imaging (MRI) are useful in accurately identifying the presence of mitral valve-septal contact, and the actual anatomical location of the mitral valve apparatus involved in SAM.

\section{Case Report}

We present a 29-year-old young male who was admitted in our institution for an elective orthopedic surgical procedure for his brachial nerve plexus injury of the right arm acquired from motor vehicular accident last 2004. He has no known comorbidities, yet prominent is his family history of early cardiac death (all less than 40 years old; no autopsies done) in his first-degree relatives, including three uncles and his father. He was asymptomatic, with stable vital signs: BP range 100 - 120/60 - $80 \mathrm{~mm} \mathrm{Hg}$, heart rate 75 - 85 beats per minute, $\mathrm{O}_{2}$ saturations $97-99 \%$ on all extremities and a body mass index of $28 \mathrm{~kg} / \mathrm{m}^{2}$. He had normal rate and regular rhythm; however, a grade 2/6 systolic murmur heard best at the apex was appreciated. The murmur increased in intensity when auscultated during standing from sitting position and when asked to do Valsalva maneuver.

Laboratory examinations revealed hemoglobin of $158 \mathrm{~g} / \mathrm{L}$, WBC of $8.34 \times 10^{9} / \mathrm{L}$, normal blood chemistry and thyroid function tests. Bleeding parameters and coagulation studies were also within acceptable limits. Chest X-ray showed 


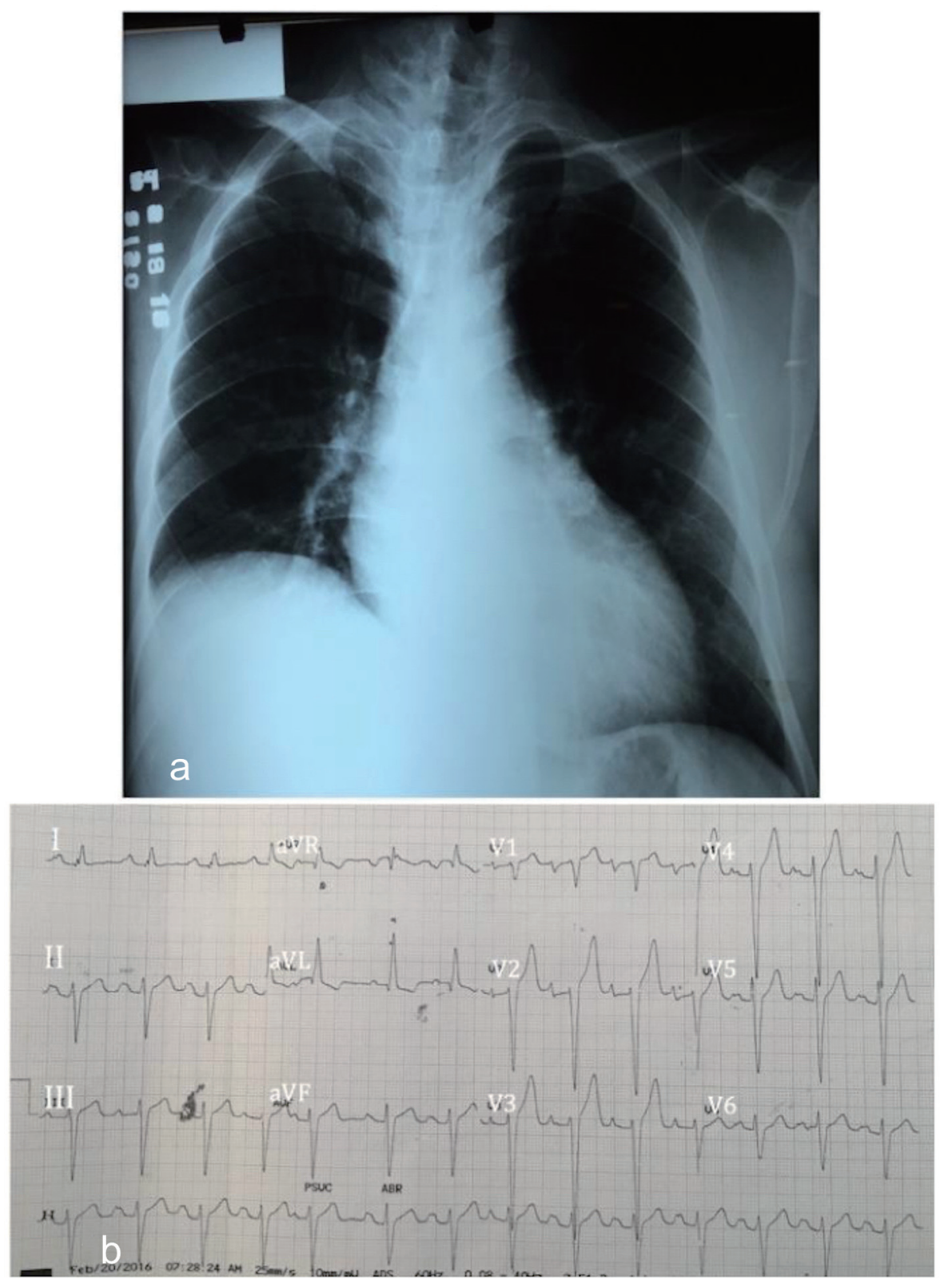

Figure 1. (a) Chest radiograph showing normal cardiac borders with loss of normal cardiac waistline suggestive of left atrial enlargement. (b) Electrocardiogram showing regular sinus rhythm, left axis deviation, left anterior fascicular block, first-degree atrioventricular block, bi-atrial abnormality and biventricular hypertrophy, isolated premature atrial complex.

blunting of the cardiac waistline suggestive of left atrial enlargement (Fig. 1a). Electrocardiogram showed regular sinus rhythm, left axis deviation $\left(-62^{\circ}\right)$, first-degree atrioventricular block and left anterior fascicular block, bi-atrial abnormality and biventricular hypertrophy with an isolated premature atrial complex (Fig. 1b).

Mitral valve prolapse with mitral regurgitation was initially considered as the pathology behind the cardiac auscultatory findings, yet an HCM with flow obstruction was a differential diagnosis. Thus, a transthoracic echocardiography (TTE) was done to evaluate valvular or other structural causes of the murmur that was heard. An asymmetrically hypertrophied left ven- tricle (septal to free wall thickness ratio of 1.6), a normal left ventricular internal dimension and a maximum left ventricular (LV) wall thickness of $33 \mathrm{~mm}$ were seen. Also prominent in the parasternal long axis is the systolic motion and contact of the anterior mitral valve and interventricular septum during early systole (Fig. 2). M-mode at the level of the mitral valve on parasternal short axis view confirmed the SAM of the anterior leaflet (Fig. 3). The exact portion of the anterior mitral valve leaflet responsible for the systolic motion was, however, not ascertained.

Continuous wave Doppler interrogation across the LVOT showed a resting peak gradient of $9 \mathrm{~mm} \mathrm{Hg}$ and mean gradi- 


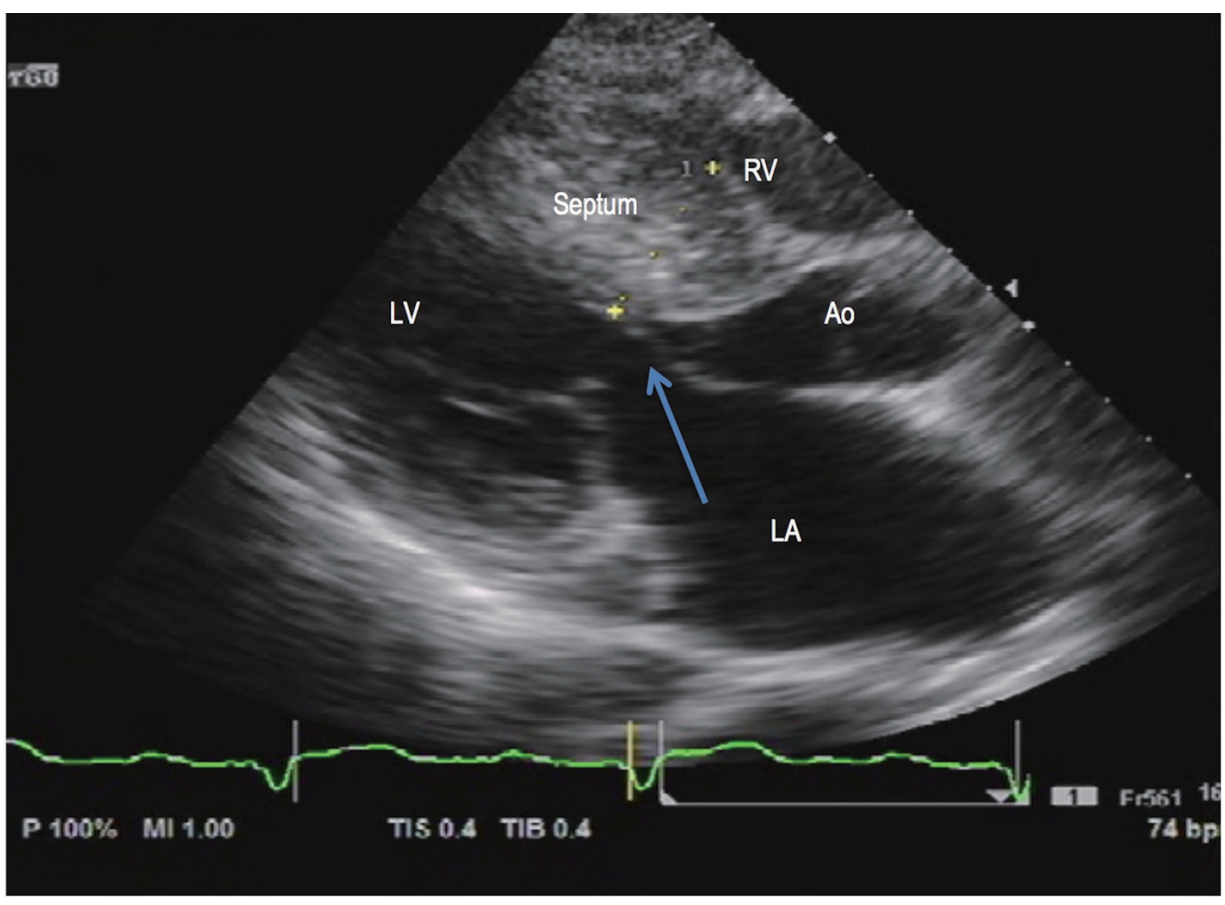

Figure 2. 2D transthoracic echocardiography. Asymmetric LV hypertrophy (septal to free wall thickness ratio of 1.6) and systolic anterior motion of the anterior mitral valve (arrow) during early systole seen on parasternal long axis view. LA: left atrium; LV: left ventricle; RV: right ventricle; Ao: aorta.

ent of $7 \mathrm{~mm} \mathrm{Hg}$ (Fig. 4a). During Valsalva maneuver, the peak gradient rose to $19 \mathrm{~mm} \mathrm{Hg}$ and mean gradient increased to $16 \mathrm{~mm} \mathrm{Hg}$ (Fig. 4b), suggestive of some, but non-significant LVOT obstruction.

The 24-h Holter monitoring was done in an attempt to detect significant arrhythmias in a setting of HCM, which might preclude us in doing a relatively invasive imaging procedure such as TEE. His Holter monitoring showed mainly sinus rhythm with only low frequency of premature atrial and ventricular complexes.

Given the paucity of clinical findings of heart failure, ideally, exercise stress echocardiography (i.e. bicycle) should confirm inducible LVOT obstruction. Short of equipment to perform such test, a TEE (Fig. 5a) was done to confirm the LVOT gradients. Likewise, TEE was done to better characterize the anatomy of the mitral valve, with particular interest on

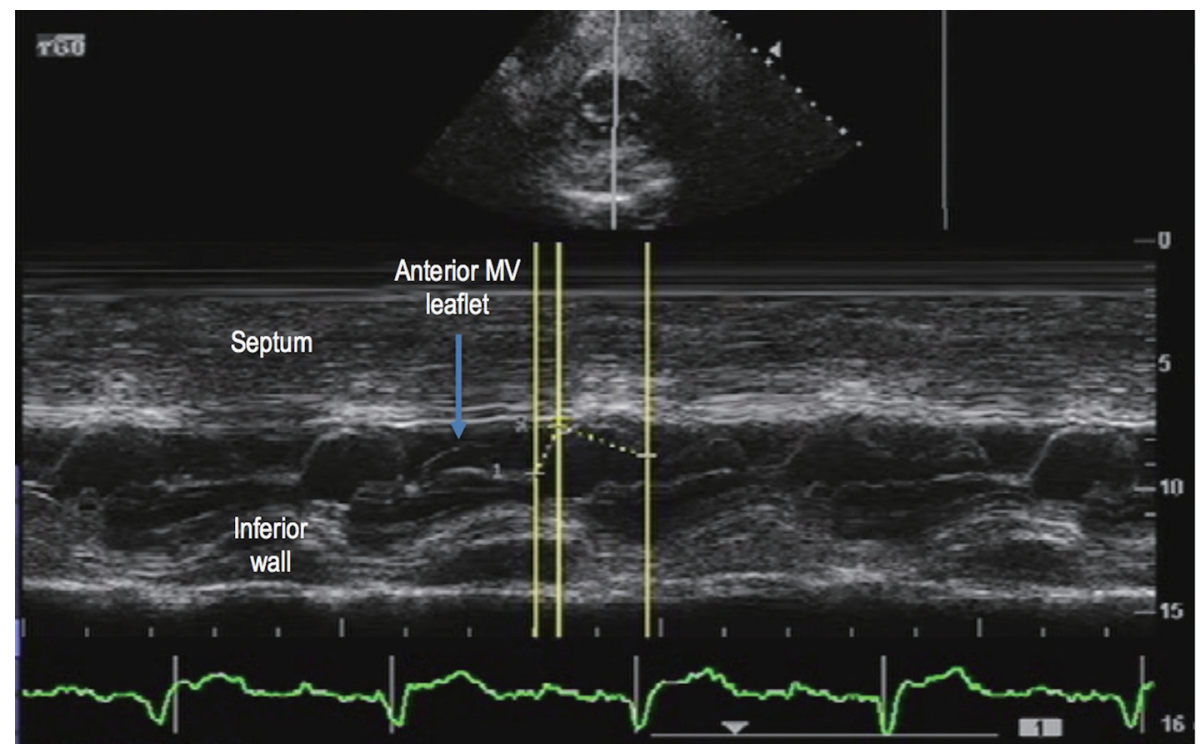

Figure 3. 2D transthoracic echocardiography. M-mode at the level of the mitral valve on parasternal short axis view confirmed systolic anterior motion (arrow) of the anterior mitral valve (MV) leaflet. 


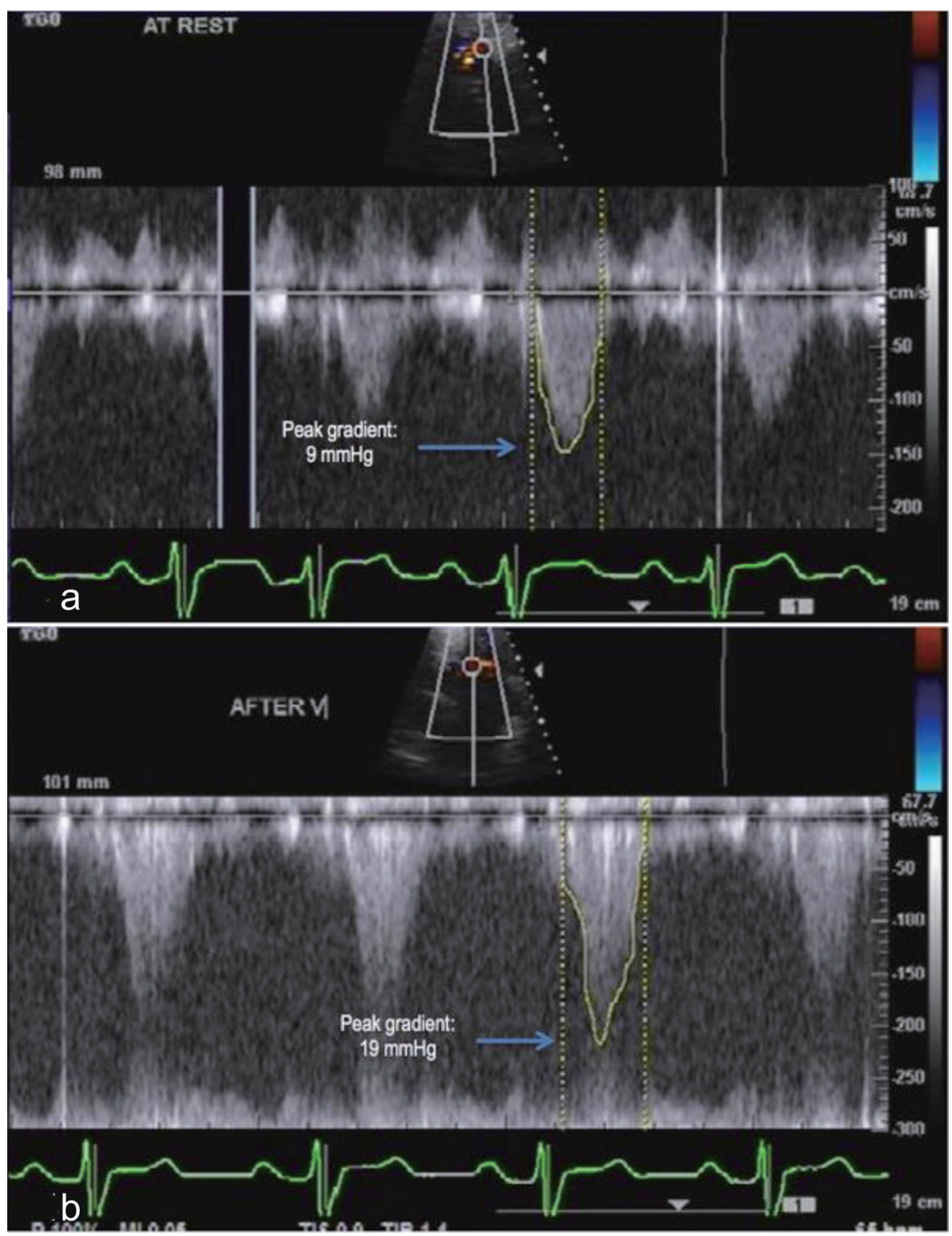

Figure 4. Continuous wave Doppler. (a) Interrogation of gradient along the LVOT revealed a peak gradient of $9 \mathrm{~mm} \mathrm{Hg}$. (b) Provocative maneuvers such as Valsalva maneuver increased the peak gradient to more than twice the gradient at rest, suggestive of some yet non-significant LVOT obstruction.

the portion of the anterior mitral valve leaflet that causes SAM. This is the mid-esophageal four-chamber view at $0^{\circ}$. The anterior mitral valve leaflet is redundant and demonstrates systolic motion mainly by its subchordal apparatus towards the interventricular septum. The anterior and posterior mitral valve leaflets are thickened with note of posterior displacement into the left atrium during systole indicative of mitral valve prolapse (Fig. 5b).

Color flow Doppler interrogation for LVOT obstruction at transgastric level long axis view, $117^{\circ}$, showed mosaic color flow across LVOT, indicative of some, though non-significant, LVOT obstruction. Likewise, a mosaic color flow was seen at the left atrium during systole, suggestive of a mild mitral regurgitation (Fig. 6). Continuous wave Doppler assessment for LVOT gradient at the level of the mitral leaflet tips revealed only a mild degree of LVOT obstruction, with mean gradient of $10 \mathrm{~mm} \mathrm{Hg}$ (Fig. 7).

The gradients across the LVOT obtained by TTE (mean gradient: $7 \mathrm{~mm} \mathrm{Hg}$ ) and that obtained during the TEE (mean gradient: $10 \mathrm{~mm} \mathrm{Hg}$ ) were more or less the same. Clinically, our patient is asymptomatic and has no heart failure symptoms, thus the echocardiographic parameters were compatible with our patient's presentation. It is worth highlighting the role of TEE which defined a redundant anterior mitral valve, which predisposed such to be dragged into the LVOT and exhibit systolic anterior motion and contribute to some yet non-significant LVOT obstruction in a setting of HCM [2]. The very mild degree of LVOT obstruction contributed by SAM primar- 


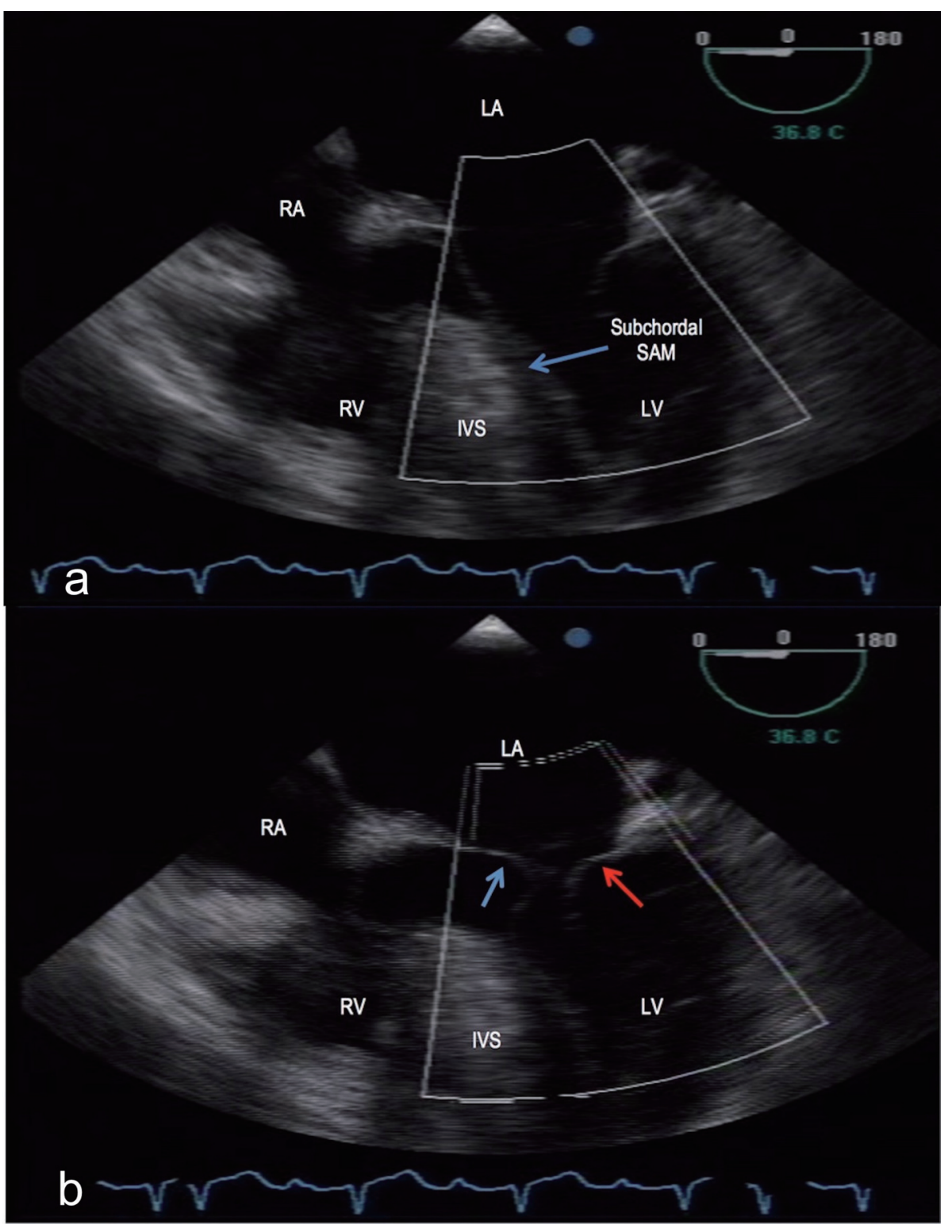

Figure 5. Transesophageal echocardiography, mid-esophageal level, $0^{\circ}$. (a) A redundant anterior mitral valve with the tip and subchordal apparatus causing systolic motion (arrow) towards a hypertrophied interventricular septum (IVS). (b) Thickened anterior (blue arrow) and posterior (red arrow) mitral valve leaflets with posterior displacement into the left atrium during systole indicative of mitral valve prolapse. LA: left atrium; RA: right atrium; RV: right ventricle; LV: left ventricle; SAM: systolic anterior motion; IVS: interventricular septum.

ily by the anterior mitral valve subchordal apparatus could be explained by the greater surface area of the chordae tendinae, hence its greater mobility [3] and lesser time for septal apposition.

Given the echocardiographic evidence and clinical diagnosis of non-obstructive HCM in an asymptomatic patient, our patient was risk stratified as having intermediate clinical risk for a low risk non-cardiac surgical procedure with no absolute contraindications, as echocardiographic features, regardless of resting outflow tract gradient, were not associated with adverse cardiac events [4].

In terms of our patient's risk for sudden cardiac death
(SCD) in a background of strong family history of early cardiac death among first-degree relatives, a thorough discussion regarding implantable cardioverter-defibrillator (ICD) for primary prevention with the patient was done. He is well aware of his risk (5.88\% risk for SCD [5], intermediate risk), and actually expressed consent. He is currently procuring funds for ICD implantation.

\section{Discussion}

$\mathrm{HCM}$ is the most common type of cardiomyopathy with ge- 


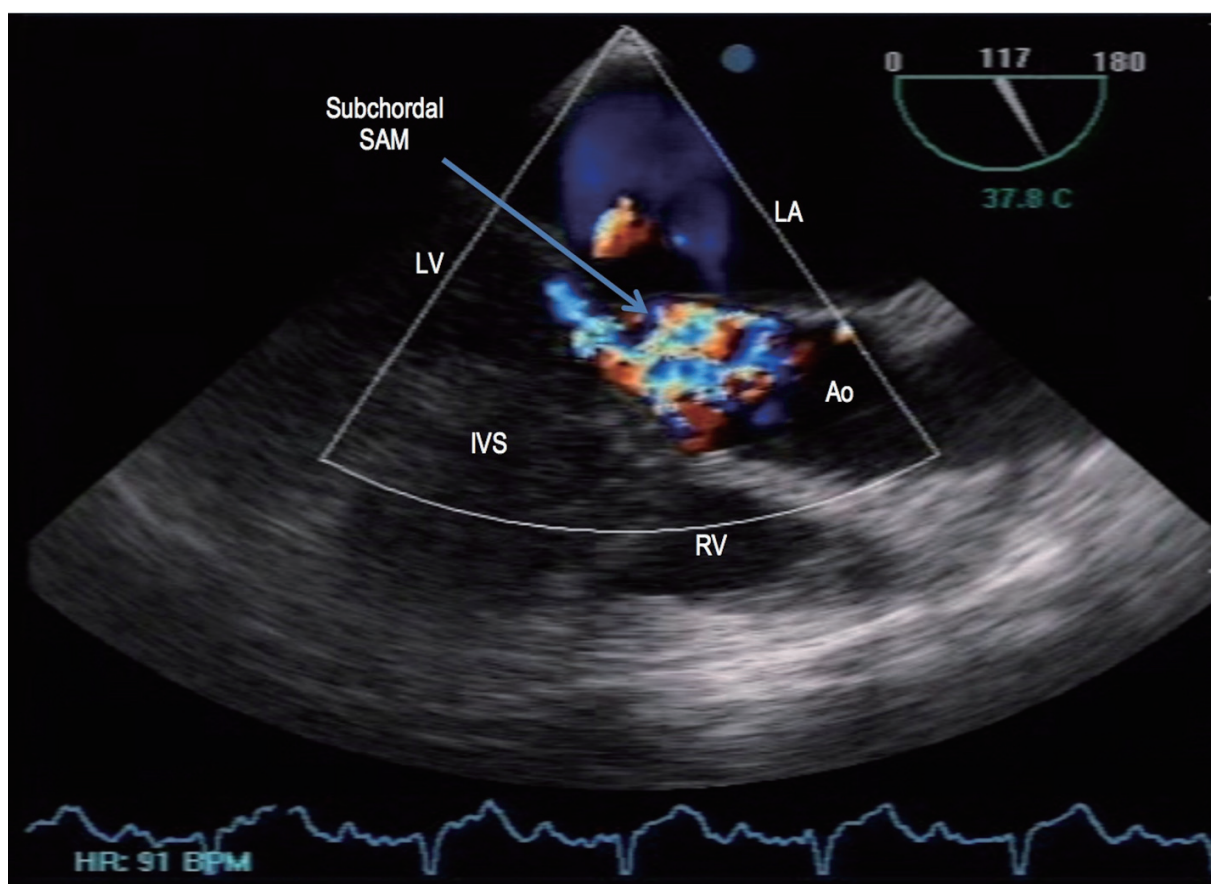

Figure 6. Transesophageal echocardiography, transgastric level, $117^{\circ}$. Mosaic color flow was seen at the level of the LVOT during systole, suggestive of some LVOT obstruction. LA: left atrium; RV: right ventricle; LV: left ventricle; Ao: aorta; IVS: interventricular septum; SAM: systolic anterior motion.

netic underlying pathology. Clinically, it is based on the demonstration of LV hypertrophy in the absence of another disease process that can reasonably explain for the magnitude of hypertrophy present. Most of the time, patients with a cardiac murmur or electrocardiographic abnormality prompting echocardiographic evaluation are incidentally diagnosed [6], such as in our patient's case.

Echocardiography remains to be an indispensable, easily accessible, relatively non-invasive and low-cost imaging modality that could be a vital tool in management of patients with HCM. Moreover, its use in detecting primary mitral valve structural abnormalities causing SAM and presence of LVOT

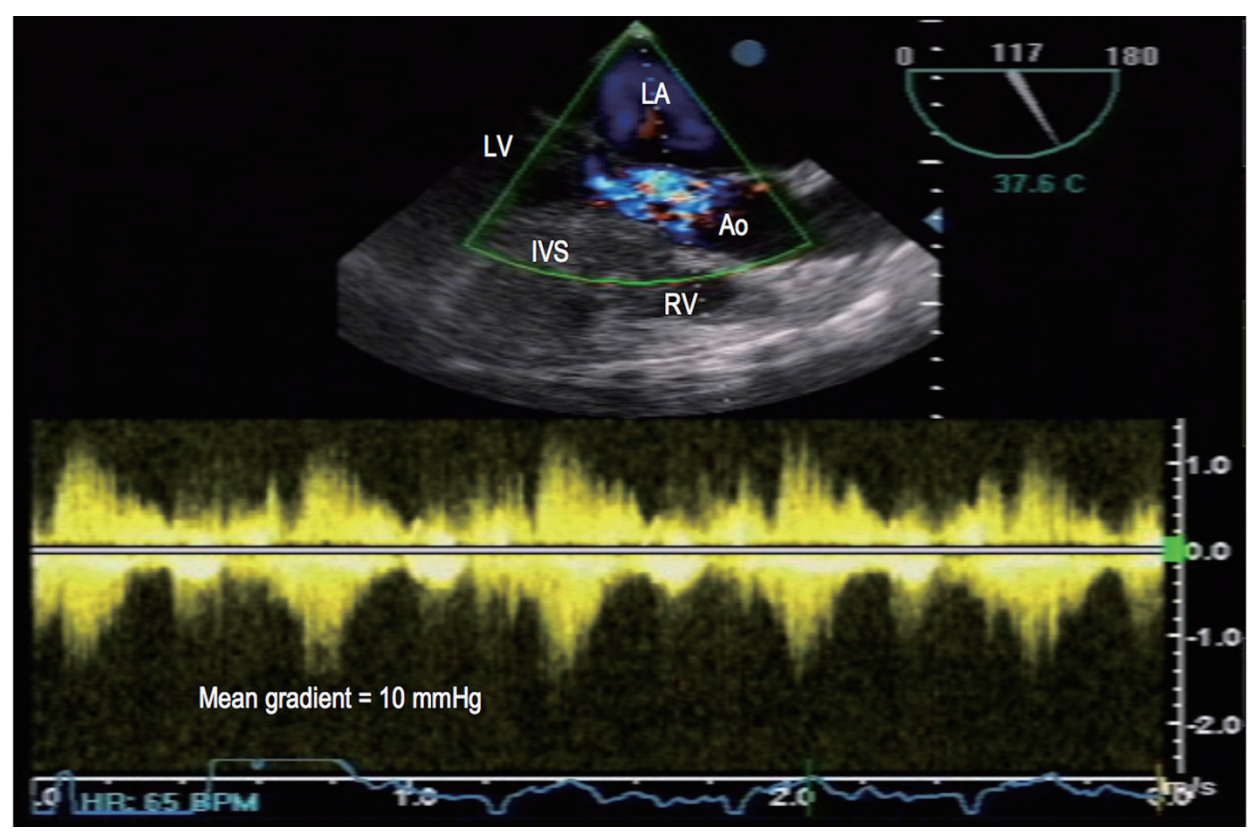

Figure 7. Transesophageal echocardiography. Continuous wave Doppler revealed a mean gradient of $10 \mathrm{~mm} \mathrm{Hg}$ across the left ventricular outflow tract (LVOT). LA: left atrium; RV: right ventricle; LV: left ventricle; Ao: aorta; IVS: interventricular septum. 
obstruction usually associated in HCM could not be overemphasized. A variety of patterns of SAM were seen in patients with HCM. Yet, available literature reporting SAM primarily by the mitral valve subchordal apparatus is very uncommon, and this study was done in 1984 which showed that out of 62 patients with HCM assessed using 2D echocardiography, in just one patient did the chordae tendineae appear to be primarily responsible for the SAM [7].

Our patient is a 29-year-old asymptomatic male who was serendipitously found on TTE with asymmetric left ventricular hypertrophy. On further non-invasive testing, for which in our case, a TEE was done, the anterior mitral valve was found to be redundant with the subchordal apparatus mainly causing SAM. Both LVOT gradients sought using TTE and TEE showed only a mild degree, non-significant LVOT obstruction, finding which was important to take note of, especially in perioperative management in non-cardiac surgery. Albeit, the aforementioned echocardiographic findings contributed in his decision to consider ICD as primary prevention for SCD.

\section{Conclusion}

To our knowledge, this is the first reported case in our country of an echocardiographic pattern of SAM primarily of the subchordal mitral valve apparatus causing some, yet non-significant, LVOT obstruction in HCM. Echocardiography, especially TEE, can be crucial in the management of patients with HCM, particularly in identifying echocardiographic features considered in the context of each patient's specific circumstances, such as in helping them decide whether an ICD is an appropriate and acceptable choice.

\section{Conflict of Interest}

The corresponding author and co-authors declare no conflict of interest related directly or indirectly in the writing of this manuscript.

\section{References}

1. Nagueh SF, Bierig SM, Budoff MJ, Desai M, Dilsizian V, Eidem B, Goldstein SA, et al. American Society of Echocardiography clinical recommendations for multimodality cardiovascular imaging of patients with hypertrophic cardiomyopathy: Endorsed by the American Society of Nuclear Cardiology, Society for Cardiovascular Magnetic Resonance, and Society of Cardiovascular Computed Tomography. J Am Soc Echocardiogr. 2011;24(5):473-498.

2. Sherrid MV, Wever-Pinzon O, Shah A, Chaudhry FA. Reflections of inflections in hypertrophic cardiomyopathy. J Am Coll Cardiol. 2009;54(3):212-219.

3. Grigg LE, Wigle ED, Williams WG, Daniel LB, Rakowski H. Transesophageal Doppler echocardiography in obstructive hypertrophic cardiomyopathy: clarification of pathophysiology and importance in intraoperative decision making. J Am Coll Cardiol. 1992;20(1):42-52.

4. Haering JM, Comunale ME, Parker RA, Lowenstein E, Douglas PS, Krumholz HM, Manning WJ. Cardiac risk of noncardiac surgery in patients with asymmetric septal hypertrophy. Anesthesiology. 1996;85(2):254-259.

5. O’Mahony C, Jichi F, Pavlou M, Monserrat L, Anastasakis A, Rapezzi C, Biagini E, et al. A novel clinical risk prediction model for sudden cardiac death in hypertrophic cardiomyopathy (HCM risk-SCD). Eur Heart J. 2014;35(30):2010-2020.

6. Maron BJ, McKenna WJ, Danielson GK, Kappenberger LJ, Kuhn HJ, Seidman CE, Shah PM, et al. American College of Cardiology/European Society of Cardiology clinical expert consensus document on hypertrophic cardiomyopathy. A report of the American College of Cardiology Foundation Task Force on Clinical Expert Consensus Documents and the European Society of Cardiology Committee for Practice Guidelines. J Am Coll Cardiol. 2003;42(9):1687-1713.

7. Spirito P, Maron BJ. Patterns of systolic anterior motion of the mitral valve in hypertrophic cardiomyopathy: assessment by two-dimensional echocardiography. Am J Cardiol. 1984;54(8):1039-1046. 